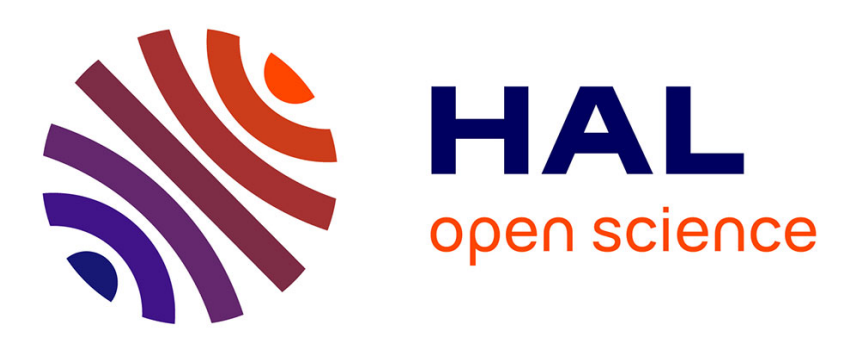

\title{
Perfectionnement d'un asservissement de fréquence, pour un spectromètre micro-onde utilisé dans l'étude de la largeur de raie de $\mathrm{SO} 2$
}

B. Lamalle, J.C. Malherbe, P. Suzeau

\section{- To cite this version:}

B. Lamalle, J.C. Malherbe, P. Suzeau. Perfectionnement d'un asservissement de fréquence, pour un spectromètre micro-onde utilisé dans l'étude de la largeur de raie de SO2. Revue de Physique Appliquée, 1981, 16 (3), pp.119-124. 10.1051/rphysap:01981001603011900 . jpa-00244901

\section{HAL Id: jpa-00244901 https://hal.science/jpa-00244901}

Submitted on 1 Jan 1981

HAL is a multi-disciplinary open access archive for the deposit and dissemination of scientific research documents, whether they are published or not. The documents may come from teaching and research institutions in France or abroad, or from public or private research centers.
L'archive ouverte pluridisciplinaire HAL, est destinée au dépôt et à la diffusion de documents scientifiques de niveau recherche, publiés ou non, émanant des établissements d'enseignement et de recherche français ou étrangers, des laboratoires publics ou privés. 


\title{
Perfectionnement d'un asservissement de fréquence, pour un spectromètre micro-onde utilisé dans l'étude de la largeur de raie de $\mathrm{SO}_{2}$
}

\author{
B. Lamalle, J. C. Malherbe et P. Suzeau \\ Laboratoire de Spectronomie de l’Université de Dijon $\left({ }^{*}\right)$, Groupe Radioélectricité, 6, boulevard Gabriel, 21100 Dijon, France
}

(Reçu le 15 juillet 1980, révisé le 6 novembre 1980, accepté le 26 novembre 1980)

\begin{abstract}
Résumé. - Un asservissement extrémal de fréquence de source micro-onde employé en spectrométrie hertzienne en bande $K_{u}$ a été étudié et réalisé. Des difficultés de précision de réponse apparaissent par manque d'un degré d'intégration lorsqu'on l'utilise en balayage linéaire de fréquence par modification des propriétés géométriques de la cavité résonnante d'analyse. Une solution simple à base d'une boucle externe non linéaire en hystérésis est proposée. La mesure du paramètre de largeur de raie de $\mathrm{SO}_{2}$ effectuée avec un spectromètre équipé de l'asservissement décrit, conduit à la valeur de $17 \mathrm{MHz}$ /torr, qui est conforme à des résultats publiés antérieurement.
\end{abstract}

\begin{abstract}
An extremal control system on frequency of a microwave source employed in microwave spectroscopy in $K_{u}$ band has been studied and realized. Some difficulties on precision response are noted by missing of one degree of integration, when used in linear frequency sweeping, by modification of the geometrical properties of the cavity cell. A simple improvement, using an external, non linear loop, with hysteresis is proposed. The measurement of linewidth parameter for $\mathrm{SO}_{2}$ obtained with a spectrometer using such a control system gives a value of $17 \mathrm{MHz} /$ torr. Which is in good agreement with results published before.
\end{abstract}

1. Introduction. - Un spectromètre hertzien mis au point au laboratoire de Radioélectricité de l'Université de Dijon emploie une cavité du type Pérot-Fabry de facteur de surtension très élevé [1]. Elle est enfermée dans une enceinte étanche dans laquelle se fait l'interaction matière-rayonnement. Sa fréquence de résonance $v_{0}$ est amenée au voisinage de la fréquence centrale de la raie d'absorption du gaz étudié. La tension $V_{0}$ provenant de la détection de l'onde en

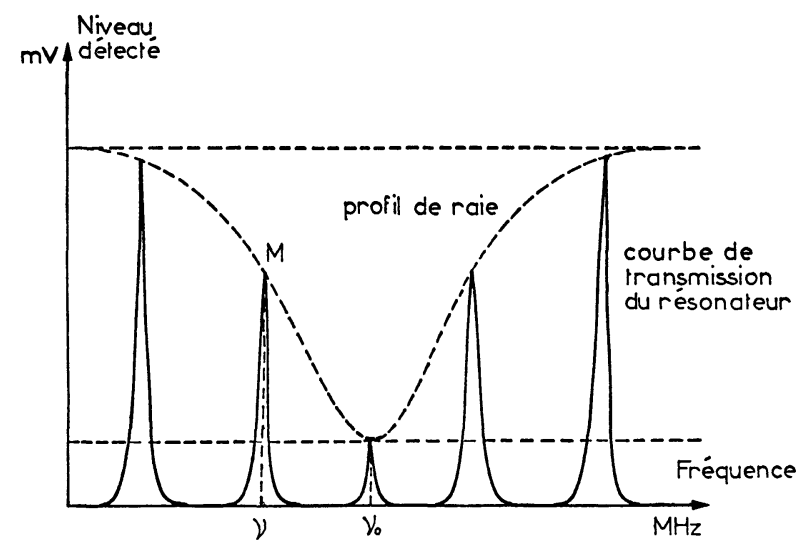

Fig. 1. - Principe du spectromètre.

[Principle of the spectrometer.]

(*) Equipe de Recherche associée au C.N.R.S. no 599. sortie de l'enceinte est proportionnelle au produit du coefficient de transmission de la cavité et du coefficient d'absorption du gaz. Elle restitue donc au cours d'un balayage en fréquence la forme de la raie avec d'autant plus de précision que la largeur de la résonance de la cavité est faible par rapport à celle de la raie (Fig. 1).

Pour cela il suffit de verrouiller la fréquence de la source sur la fréquence de résonance de la cavité d'une part, et d'autre part de faire varier les dimensions géométriques de la cavité. Ce qui est fait par le déplacement d'un des miroirs.

La figure 2 donne le schéma général du spectromètre.

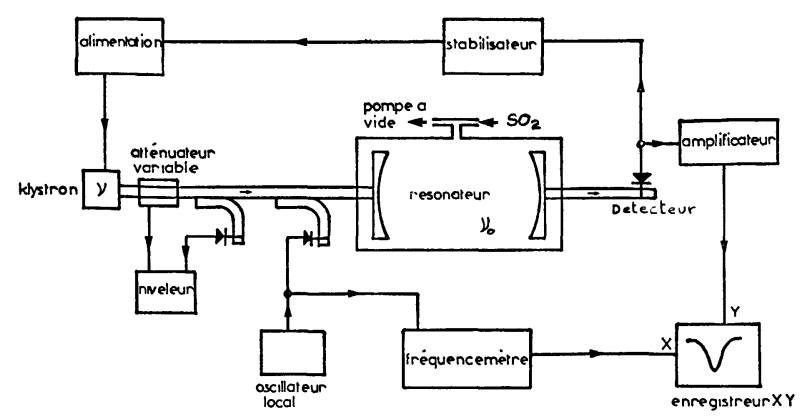

Fig. 2. - Schéma général du spectromètre.

[Block diagram of the spectrometer.] 
2. Etude du stabilisateur de fréquence. -2.1 Principe. - L'asservissement de la fréquence de la source sur celle de la cavité est réalisé par un stabilisateur de type Jung $[2,3]$ dont le principe est le suivant. Une tension de modulation $\Delta V_{\mathrm{m}}$ (démarche) sinusoïdale de pulsation $\omega$ est superposée à la tension de réflecteur de klystron. Il en résulte une modulation de fréquence de la source de profondeur $\Delta v_{\mathrm{m}}$. La cavité joue le rôle de discriminateur de fréquence si les fréquences $v_{0}$ de résonance de la cavité et $v_{\mathrm{s}}$ de la source en absence de correction sont voisines. On recueille sur le détecteur un signal dont le premier harmonique (de pulsation $\omega$ ) a une amplitude $V_{1}$ et une phase $\varphi$ fonction de la position relative de $v_{0}$ et $v_{\mathrm{s}}$. Ce signal amplifié, délivre après détection synchrone une tension $V_{\mathrm{c}}$ (dite de correction) qui, une fois filtrée est ajoutée à la tension de réflecteur $\mathrm{du}$ klystron de manière à minimiser la différence $v_{\mathrm{s}}-v_{0}$.

On rappelle la relation fondamentale de ce type d'asservissement

$$
\Delta v_{\mathrm{c}}=\left(\Delta v_{\mathrm{s}}+G \Delta v_{0}\right) /(1+G)
$$

$-\Delta v_{\mathrm{c}}$ et $\Delta v_{\mathrm{s}}$ sont les fluctuations de la source respectivement en boucle fermée et en absence de correction.

$-\Delta v_{0}$ mesure les variations de la fréquence de résonance de la cavité, $G$ enfin est le gain total de la boucle. Il est de la forme $G=K_{\mathrm{s}} \cdot K_{\mathrm{R}} \cdot K_{\mathrm{D}}$.

- $K_{\mathrm{s}}$ est le gain du stabilisateur pour un réglage correct de la phase, il est légèrement inférieur au gain de l'amplificateur de signal.

$-K_{\mathrm{D}}$ est le gain du discriminateur de fréquence. Il est proportionnel à $Q^{2}$ ( $Q$ est le coefficient de surtension de la cavité), aux coefficients de couplage et enfin au rendement du détecteur et à la puissance du signal émis.

- $K_{\mathrm{R}}$ est la pente de l'organe de modulation de fréquence. Ici c'est l'électrode réflecteur d'un klystron. Il est toujours de l'ordre de $1 \mathrm{MHz} / \mathrm{V}$.

Nous terminons ce rapide rappel théorique par le filtre passe-bas dont le double rôle est de filtrer la tension en sortie du démodulateur synchrone et d'assurer la stabilité de la boucle. De la condition de filtrage on tire l'inégalité $\omega>G / \tau$ avec $\tau=R C$.

2.2 Choix Des Paramètres. - Pour faire un relevé très précis de profil de la raie nous voulions réaliser un stabilisateur plus précis et plus rapide que les modèles existants [4]. Pour cela nous nous sommes appliqués à optimiser point par point chaque élément d'un stabilisateur classique.

Tout d'abord le compromis entre le gain $G$ et le temps de réponse $\tau$ de l'asservissement sera d'autant meilleur que $\omega$ sera grand. La valeur choisie $\omega / 2 \Pi=100 \mathrm{kHz}$ nous apparait être la limite supérieure de détectabilité des diodes qu'elles soient classiques ou Schottky. Ensuite notre choix s'est arrêté sur les valeurs $G<300$ et $\tau=3 \mathrm{~ms}$.

Ce gain est très élevé, nous avons pu l'obtenir en utilisant d'une part une cavité de surtension très élevée $Q=3 \times 10^{5}$ et d'autre part des diodes Schottky nettement supérieures en bande $K_{u}$ aux diodes classiques. Et enfin un amplificateur de signal faible bruit très performant, de gain réglable électriquement supérieur à $100 \mathrm{~dB}$ et de bande passante étroite. Cette sélectivité nous a obligé par ailleurs à utiliser une référence très stable réalisée par divisions successives de fréquence en partant d'un quartz à $5 \mathrm{MHz}$.

Il nous était par ailleurs impossible d'agir ni sur la puissance de la source imposée par les conditions expérimentales, ni sur le couplage de la cavité pour ne pas détériorer sa surtension.

Le système réalisé délivre une tension de correction limitée à $\pm 10 \mathrm{~V}$.
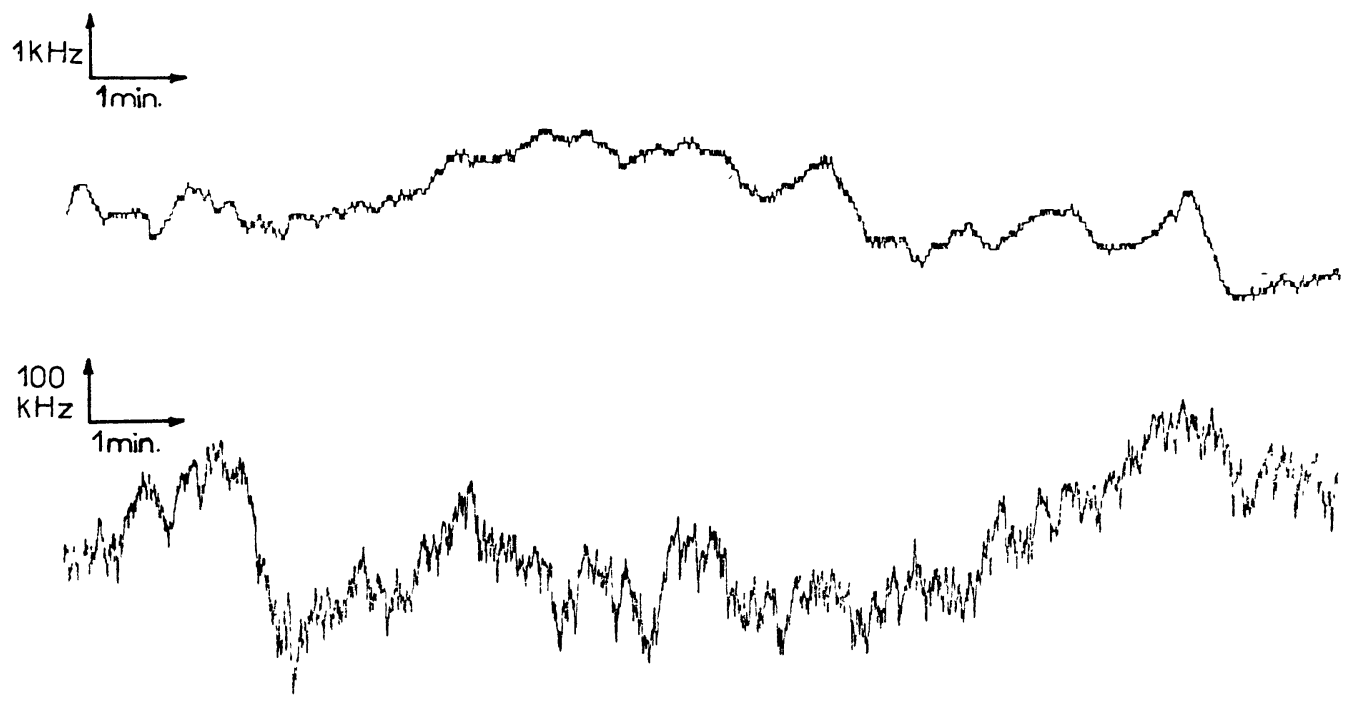

Fig. 3. - Courbes de stabilité statique.

[Plot of the static stability.] 
2.3 Performances StatiQues. - Les fréquences $v_{\mathrm{s}}$ du klystron libre et $v_{\mathrm{c}}$ asservi ont été enregistrées successivement pendant $10 \mathrm{~min}$. avec des échelles différentes (Fig. 3). On vérifie ainsi que les performances recherchées ont été atteintes. En particulier que la précision de la fréquence de l'ordre de $3 \mathrm{kHz}$ pendant 10 min. est suffisante pour étudier le profil de la raie. Elle est meilleure que $10^{-7}$ à $33 \mathrm{GHz}$.

3. Etude dynamique. - L'appareil précédemment décrit stabilise convenablement une source hyperfréquence sur une cavité fixe. En spectronométrie hertzienne il a permis de bonnes mesures point par point. Par contre lorsqu'on fait varier la fréquence $v_{0}$, ce qui revient à soumettre l'asservissement à une rampe de consigne, on observe une croissance de l'erreur avec cette consigne. La figure 4 donnant la courbe de variation de $v_{\mathrm{c}}$ en fonction de $V_{\mathrm{c}}$ (en ayant veillé à prendre une erreur de position minimale au départ) rend compte de ce phénomène. Cet enregistrement a été réalisé avec une vitesse de balayage de $v_{0}$ très faible, de l'ordre du $\mathrm{MHz} /$ minute. Pour les faibles valeurs de $V_{\mathrm{c}}$ (dans l'intervalle $-1,+1 \mathrm{~V}$ ) on a approximativement une droite dont la pente est légèrement inférieure à celle du klystron $(1 \mathrm{MHz} / \mathrm{V}$ sur le réflecteur), pour les plus grandes valeurs on a une forte courbure de la représentation.

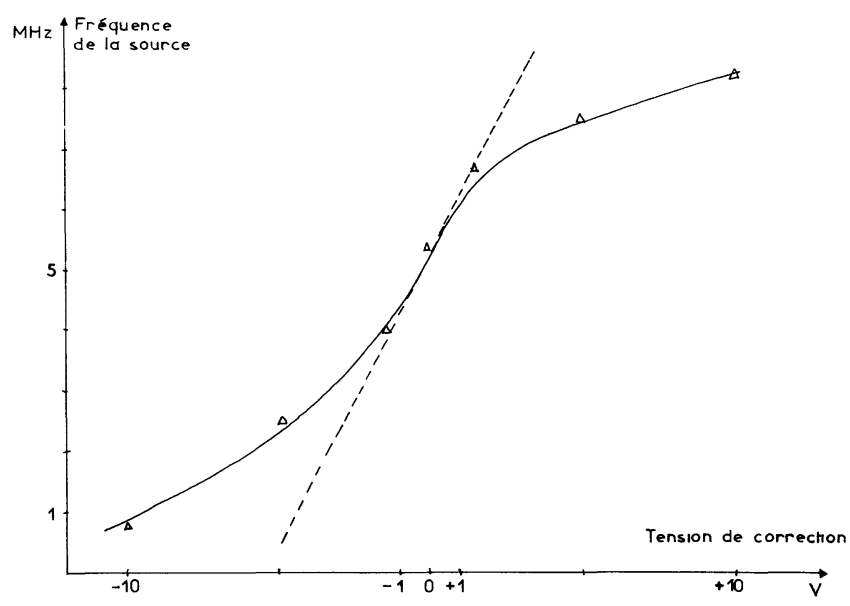

Fig. 4. - Courbe de réponse dynamique.

[Plot of the dynamical response.]

L'accroissement constant de l'erreur pour les faibles valeurs de $V_{\mathrm{c}}$ s'explique facilement par le nombre insuffisant de pôles pour le gain en boucle ouverte. La courbure obtenue pour les plus grandes valeurs de $V_{\mathrm{c}}$ est quant à elle due principalement à la nonlinéarité du discriminateur hors de sa zone centrale. Non-linéarité qui, entraînant une réduction rapide du gain, accroît par conséquent l'erreur de manière inacceptable.

Pour permettre l'emploi de cet appareil en dynamique, il faut donc munir sa chaîne d'une ou plu- sieurs intégrations, tout en maintenant l'erreur résiduelle dans la zone de linéarité du discriminateur. On sait en effet que pour annuler l'erreur en régime stationnaire il faut une intégration pour une consigne constante et deux intégrations pour une consigne en forme de rampe. Il faudrait donc introduire dans notre cas un terme en :

$$
\frac{1}{p^{K}} \quad \text { avec } K \geqslant 1 .
$$

Pour $K=1$ on aurait une erreur constante et pour $K \geqslant 2$ une erreur nulle [5].

Ceci rejoint notamment une des conditions requises pour la réponse pile d'un système échantillonné [6]. $\mathrm{Sa}$ fonction de transfert doit comporter au moins $(m-n)$ intégrations, où $m$ représente le degré du polynôme de consigne (rampe $m=1$ ) et $n$ l'ordre de l'extrapolateur (ici ordre 0 pour le détecteur synchrone).

Il n'est pas toujours aisé d'introduire un élément d'intégration dans la chaîne. Notamment à cause de problèmes apparaissant à la mise en service, et surtout de ce qu'il faut ajuster la constante de temps $\mathrm{du}$ circuit correspondant pour chaque vitesse de balayage en fréquence. Une autre solution consiste à introduire une non-linéarité présentant en dehors de sa zone de saturation un très grand gain $[7,8]$.

Un plus ou moins idéal peut répondre à ce besoin puisque son gain équivalent étudié par la méthode du premier harmonique est :

$$
N=\frac{4 V_{\mathrm{so}}}{\Pi V_{\mathrm{em}}}
$$

où $V_{\text {so }}$ est l'amplitude de sortie du plus ou moins et $V_{\text {em }}$ l'amplitude d'un signal sinusoïdal appliqué à l'entrée de la non-linéarité.

Ce gain tend théoriquement vers l'infini pour les plus petites valeurs de $V_{\mathrm{em}}$. On a alors une oscillation limite stable dont l'amplitude doit être rendue compatible avec l'erreur maximale tolérée.

Dans notre cas, afin d'apporter une solution aussi simple que possible et de ne pas modifier un appareil existant, le problème a été résolu au moyen d'une boucle supplémentaire, externe. Cette boucle comprend un plus ou moins à hystérésis, associé à un motoréducteur agissant sur le potentiomètre de réglage de la tension du réflecteur du klystron (Fig. 5).

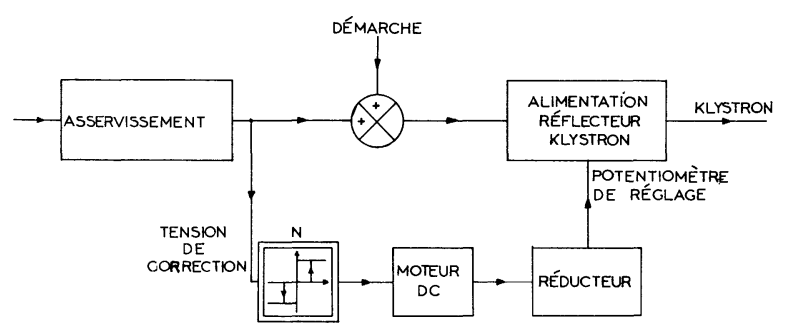

Fig. 5. - Schéma de principe de la boucle externe.

[Block diagram of the external loop.] 
On a choisi pour la non-linéarité $N$ un plus ou moins à double hystérésis dont la largeur entre seuil est double de la largeur de l'hystérésis plutôt qu'un plus ou moins idéal (Fig. 6) pour les raisons suivantes :

a) Cette non-linéarité évite les inversions brutales du sens de rotation du servomoteur.

b) Elle évite également les risques technologiques d'erreur de signe de la correction.

c) Elle permet le réglage de l'oscillation limite.

Le module et l'argument d'une telle non-linéarité analysée par la méthode du premier harmonique ont respectivement pour expression :

$$
\begin{aligned}
N & =\frac{2 \sqrt{2} V_{\mathrm{sm}}}{\Pi V_{\mathrm{c}}} \sqrt{1+\sqrt{1-\left(\frac{V_{\mathrm{B}}}{V_{\mathrm{c}}}\right)^{2}}} \\
\operatorname{Arg} N & =-\frac{1}{2} \operatorname{Arc} \operatorname{Sin} \frac{V_{\mathrm{B}}}{V_{\mathrm{c}}} .
\end{aligned}
$$

De la relation 4 on tire que le gain équivalent est maximum pour une valeur de $V_{\mathrm{c}}$ très voisine de $V_{\mathbf{B}}$ $\left(1,06 V_{\mathrm{B}}\right)$.

La relation (5) montre que le déphasage maximum est de $-45^{\circ}$ ce qui se traduit dans notre application par un effet de retard d'autant plus important que $V_{\mathrm{B}} / V_{\mathrm{c}}$ est proche de 1 par valeurs inférieures.

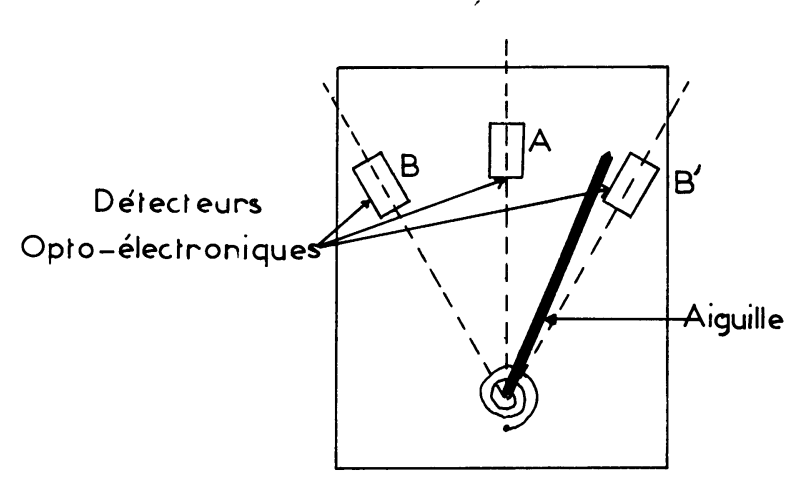

a)

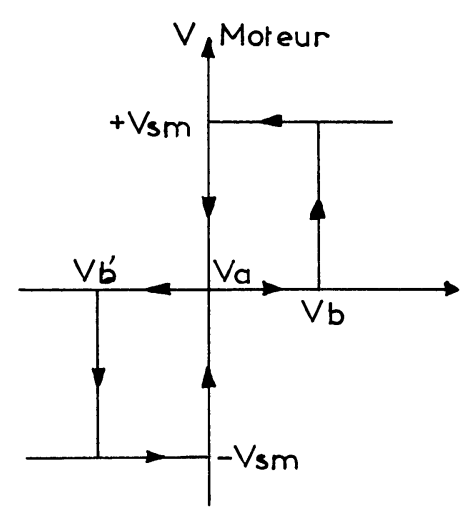

b)

Fig. 6. - a) Détecteur trois positions. b) Représentation de l'hystérésis.

[a) Three positions detector. b) Representation of the hysteresis.]
En diminuant le rapport $V_{\mathrm{B}} / V_{\mathrm{c}}$, c'est-à-dire en augmentant la sensibilité du système, on réduit donc ce retard. On vérifie en outre que pour ce type de nonlinéarité dont le seuil est égal à la largeur de l'hystérésis l'oscillation limite est stable. Cette oscillation limite stable a une période et une amplitude qui diminue lorsque la sensibilité augmente.

Cette non-linéarité a été obtenue simplement en mesurant la tension de correction au moyen d'un galvanomètre à zéro central dont on détecte trois positions différentes de l'aiguille au moyen de capteurs optoélectroniques (Fig. 6a). Un montage à circuits logiques et relais commande la mise en marche du moteur dans un sens lorsque l'aiguille atteint le capteur B, dans l'autre sens quand elle atteint le capteur $\mathrm{B}^{\prime}$, arrête le moteur (quel que soit le sens) lorsqu'elle atteint le capteur A (tension de correction nulle) (Fig. 6b).

Le galvanomètre étant porté à un potentiel flottant élevé une bonne isolation s'impose et l'aiguille doit être en matière isolante.

La figure $7 a$ représente l'amplificateur de gain maximum 4 associé au galvanomètre à zéro central.

L'entrée reçoit la tension de correction. Le potentiomètre $\mathrm{P}_{1}$ permet le réglage de la sensibilité de l'ensemble.

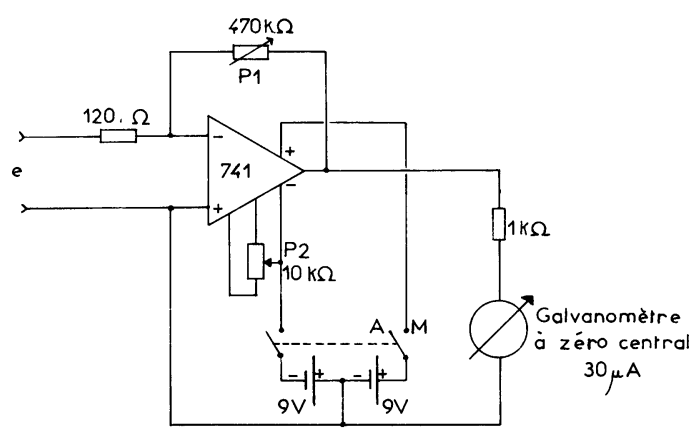

a)

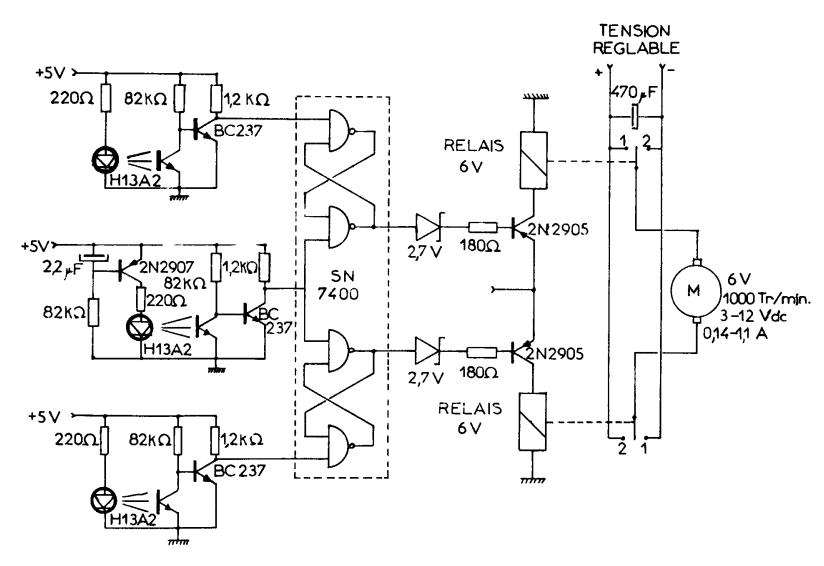

b)

Fig. 7. - a) Circuit de commande du galvanomètre. b) Circuit logique associé aux capteurs.

[a) Galvanometer driver. b) Logical circuitry associated to transduceurs.] 
Le potentiomètre $\mathrm{P}_{2}$ assure le réglage du zéro. L'ensemble est alimenté en flottant par deux piles de $9 \mathrm{~V}$ en raison du potentiel élevé du circuit par rapport à la masse $(\simeq 3 \mathrm{kV})$.

La figure $7 b$ représente la logique associée aux capteurs optoélectroniques $\mathrm{A}, \mathrm{B}$ et $\mathbf{B}^{\prime}$. Une remarque s'impose sur le circuit du capteur A. Lors de la mise en service, l'aiguille du galvanomètre étant dans une position quelconque, il convient que le moteur soit à l'arrêt. Ceci est obtenu grâce à une alimentation retardée de la diode électroluminescente $\mathrm{A}$ par un circuit RC.

L'erreur maximale de calage du klystron par rapport à la cavité résonnante obtenue avec ce dispositif est de l'ordre de $4 \mathrm{kHz}$ pour le gain maximum. La largeur à mi-hauteur de la résonance de la cavité étant mesurée à $100 \mathrm{kHz}$, l'approximation parabolique du sommet conduit à une estimation de l'erreur d'amplitude inférieure à $0,5 \%$ ce qui est suffisant pour les mesures physiques à effectuer.

4. Exploitation expérimentale du dispositif. - A l'aide du spectromètre amélioré par le dispositif non linéaire décrit précédemment on a pu étudier la transition $29_{6,24} \rightarrow 30_{5,25} \mathrm{de}^{32} \mathrm{SO}_{2}$ à $36338,05 \mathrm{MHz}$.

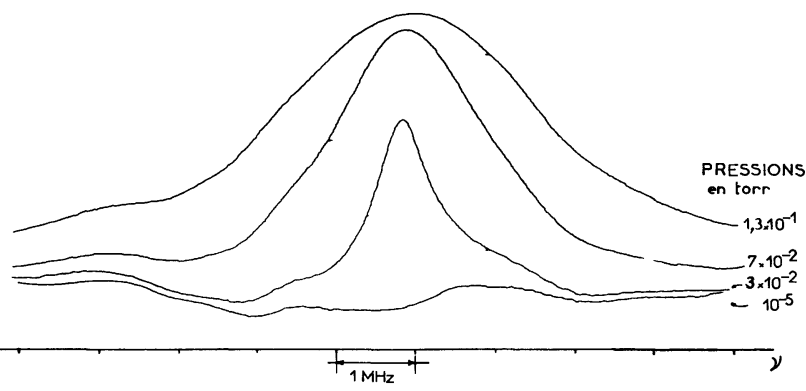

a)

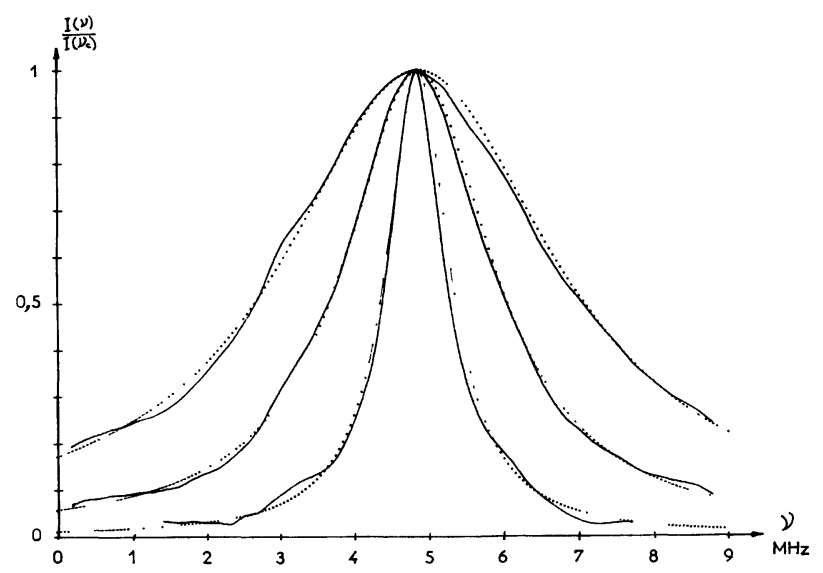

b)

Fig. 8. $-a$ ) Raie $29_{6,24} \rightarrow 30_{5,25}$ de $\mathrm{SO}_{2}$ pour différentes pressions. b) Enregistrements $I(v) / I\left(v_{\mathrm{c}}\right)$ (traits pleins) et raies théoriques (pointillés).

[a) Transition $29_{6,24} \rightarrow 30_{5,25}$ of $\mathrm{SO}_{2}$ for different pressures. b) $I(v) / I\left(v_{\mathrm{c}}\right)$ calibrated records (continuous line) and theorical lines (dotted line).]
On a réalisé une série de trois enregistrements de cette raie d'absorption à des pressions de $1,3 \times 10^{-1}$, $7 \times 10^{-2}$ et $3 \times 10^{-2}$ torr. Un enregistrement est également fait en absence de gaz à une pression de l'ordre de $10^{-5}$ torr (Fig. 8a).

L'exploitation des résultats faite à l'aide d'un calculateur Hewlett Packard HP 9815 A associé à une table traçante, selon un processus décrit en référence [1] nous permet de tracer directement les intensités relatives des raies $I(v) / I\left(v_{\mathrm{c}}\right)\left(v_{\mathrm{c}}\right.$ est la fréquence centrale de la raie) (Fig. $8 b$ ). La figure 9 montre que la variation de $\Delta v$ en fonction de la pression est une droite. La pente de cette droite donne le paramètre de largeur de raie $(\Delta v / p)$. On a obtenu :

$$
\Delta v / p=17 \mathrm{MHz} / \text { torr } .
$$

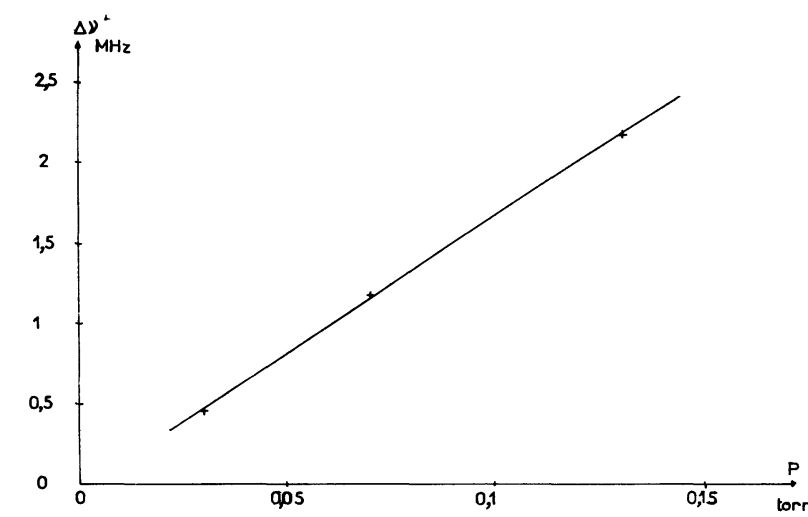

Fig. 9. - Variation de la demi-largeur à mi-hauteur en fonction de la pression.

[Half-widht versus pressure.]

Cette détermination est en parfait accord avec les résultats publiés antérieurement $[9,10]$.

Pour justifier la valeur du paramètre on a reconstitué le profil de la raie suivant la théorie d'Anderson. A une pression $p$, les formes lorentziennes sont données par [11]

$$
I(v)=\frac{A \Delta v}{\left(v-v_{\mathrm{c}}-a \Delta v\right)^{2}+(\Delta v)^{2}}
$$

$A$ et $a$ sont des constantes. Ici aucun shift n'ayant été observé $a=0$ soit

$$
I(v)=\frac{A \Delta v}{\left(v-v_{\mathrm{c}}\right)^{2}+(\Delta v)^{2}} .
$$

D'où l'intensité relative

$$
\frac{I(v)}{I\left(v_{\mathrm{c}}\right)}=\frac{\left(\frac{\Delta v}{p} \times p\right)^{2}}{\left(v-v_{\mathrm{c}}\right)+\left(\frac{\Delta v}{p} \times p\right)^{2}} .
$$

La figure $8 b$ permet de constater le bon accord entre les profils expérimentaux et calculés. 
5. Conclusion. - Dans cet article un dispositif d'asservissement de fréquence de source micro-onde sur la fréquence de résonance de la cavité d'analyse d'un spectromètre hertzien a été présenté. Ses performances statiques sont meilleures que celles des dispositifs antérieurs. En dynamique il a fallu lui apporter une modification au moyen d'une boucle externe non linéaire pour améliorer le comportement du système lorsqu'on balaye linéairement la fréquence. Des résultats expérimentaux obtenus avec ces appareils illustrent par leur conformité avec des résultats publiés antérieurement le bon fonctionnement de l'asservissement.

\section{Bibliographie}

[1] Mathelin, J. P., Suzeau, P., Lamalle, B., Can. J. Phys. 56 (1978) 882

[2] JUng, P., J. Sci. Instrum. 37 (1960) 372.

[3] SERE, D., Thèse Doctorat 3e cycle, Paris (1965).

[4] Miller, NDJ., J. Phys. E 9(1976).

[5] VIDAL, P., Aide-mémoire d'Automatique (Dunod-Paris) (1978).

[6] Sevely, Y., Systèmes et asservissements linéaires échantillonnés (Dunod Université, Paris) (1973).

[7] Lhote, F., Séminaire d'automatique club EEA. Champagnyen-Vanoise (1980).
[8] Decaulne, P., Gille, J. Ch., Pelegrin, M., Introduction aux systèmes asservis extrémaux et adaptifs Dunod Automatique-Paris (1976).

[9] Kolbe, W. F., Leskovar, B., Buscher, H., J. Mol. Spectrosc. 59 (1976) 86.

[10] Meier, D., J. Quant. Spectrosc. Radiat. Transfer. 19 (1978) 323.

[11] Townes, C. H., Schawlon, A. L., Microware spectroscopy (Mc Graw-Hill Book Co., New York) 1955. 